\title{
Social and environmental adversities, neurobiological processes and mental disorders
}

\author{
C. Barbui, Editor* and B. Saraceno, Guest Editor
}

First published online 11 January 2016

Key words: Mental disorders, neurobiology, environment.

In this Issue of Epidemiology and Psychiatric Sciences Nikolas Rose provides a thought-provoking analysis of the relationship between clinical psychiatry and neurobiological research (Rose, 2015). The idea that mental disorders are disorders of brain circuits, and that the brain will eventually provide an objective basis for establishing a causal relationship between altered brain circuits, psychiatric symptoms, diagnostic features and treatment options, is described as a reductionist approach to the role of neurobiology in psychiatry. The unshakeable idea that 'the brain holds the key' Nikolas Rose argues - has negative consequences: in terms of diagnostic categories, as when the Diagnostic and Statistical Manual, Edition 5 (DSM 5) was published there was not a single clinically validated biomarker for any psychiatric disorder; in terms of explanatory models, as we are a long way from identifying neural bases of mental disorders; in terms of pharmacological treatments, as few now believe in the basic explanatory form of neurotransmitter hypothesis of mental disorder.

The Editorial then makes a proposal from which substantial progress might be made (Rose, 2015). Mental disorders should not be considered brain disorders but disorders of persons 'always in transaction with a social and environmental setting'. These transactions may shape neurobiology across the life-span and should always be included in our conceptual models, surpassing the limits of, quoting Leon Eisenberg, a 'brainless' or 'mindless' psychiatry, which have either oriented exclusively to models excluding biological influences, or to models excluding psychological ones (Eisenberg, 1986). Sociological and epidemiological studies have consistently shown correlations between diagnoses of mental disorder and a whole range of social and environmental adversities. Therefore,

* Address for correspondence: Professor C. Barbui, Department of Public Health and Community Medicine, Section of Psychiatry, Ospedale Policlinico 'G.B. Rossi', Piazzale L.A. Scuro 10, 37134 Verona, Italy.

(Email: corrado.barbui@univr.it) adversities might affect neurobiological mechanisms and processes that might ultimately lead to mental disorders. Clearly, we do not know how adversities 'get under the skin', but Nikolas Rose describes some possible mechanisms (Rose, 2015).

If you think this is too theoretical and difficult to translate into something real then you should read a second Editorial published by Carmine Pariante in the same Issue of Epidemiology and Psychiatric Sciences (Pariante, 2015). It gives a bright example of psychopathology arising outside the brain because of environmental factors. Carmine Pariante shows how social adversities, including exposure to personal maltreatment or violence, socioeconomic disadvantage or isolation, are factors associated with an increased immune activation, and this, for example high levels of inflammation, may predict future development of psychopathology. In experimental models, it is also possible to induce psychiatric symptoms administering an immune activator. This would suggest that transactions with adversities may shape neurobiological mechanisms, with a peripheral localisation, that may ultimately lead to neuropsychiatric symptoms such as depression, anxiety, or psychotic symptoms. Additionally, the evidence that adding an antiinflammatory to an antidepressant or an antipsychotic increases the efficacy of these medications - Carmine Pariante argues - is another piece of evidence in favour of a link between these factors (Pariante, 2015).

As practicing doctor (C. B.) and policy expert (B. S.), we argue that these two Editorials, with the example of immune-related mechanisms as intermediate element between social factors and mental disorders, prompt the following considerations.

- The practical relevance of this model depends on its epidemiology. Which is the epidemiological fraction of people with, say for example, depression and high levels of inflammation? Is this immune-related mechanism present in the majority, minority or in a negligible proportion of every-day individuals 
seeking care for depression under ordinary circumstances? Apart from the heuristic value of the model, which is remarkable, its practical value may depend on its epidemiological impact.

- The efficacy of anti-inflammatory medications should probably be studied in the subgroup of patients with depression and high baseline inflammatory biomarkers. So far, no clinical trials have been conducted in this patient population. This would make sense as the expectation is to detect an anti-depressant effect mainly in patients who have higher levels of inflammation at study entry. Is this population (patients with depression and high inflammatory biomarkers) easily defined? Is there consensus among experts on who patients with depression and high inflammatory biomarkers are? This is clinically relevant, as doctors would need a clear and unambiguous way of identifying cases with 'increased inflammation'.

- Perhaps people with post-traumatic stress disorder (PTSD) and acute stress disorder (ASD) may represent a more homogeneous patient population where the effects of anti-inflammatory medications might be studied. Noteworthy, it has recently been shown that hydrocortisone, given within the first month after a traumatic or aversive event, showed a large effect to prevent PTSD or ASD (Sijbrandij et al. 2015). As individuals at risk for PTSD show lower cortisol concentrations shortly after trauma, and low concentrations of circulating cortisol are assumed to prolong the acute adrenergic response to the traumatic event, early administration of glucocorticoids might be expected to counteract this maladaptive process.

- Proving a relationship between social factors and mental disorders through immune-related mechanisms might be seen as another strong argument in favour of the implementation of policies aimed at preventing mental disorders and promoting mental health. We now have remarkable research findings consistently showing that modifiable conditions, such as child abuse, are risk factors for many mental disorders, and the case for action in primary prevention has been gaining solid scientific foundations thanks to gene-environment $(\mathrm{G} \times \mathrm{E})$ studies, with contributions to both risk and resilience factors (Levav \& Saraceno, 2014). If social adversities affect neurobiological mechanisms and processes that might ultimately lead to mental disorders, not only neurobiological mechanisms should be 'corrected', but also social adversities should be neutralised. These concepts have recently been emphasised by a thematic paper promoted by WHO and the Gulbenkian foundation which showed that certain population subgroups are at higher risk of mental disorders because of greater exposure and vulnerability to unfavourable social, economic and environmental circumstances, interrelated with gender (World Health Organization and Gulbenkian Foundation, 2014). WHO and the Gulbenkian foundation strongly suggests policy actions to mitigate the effects of these adversities, beginning before birth and progressing into early childhood, older childhood and adolescence, during family building and working ages, and through to older age.

\section{Acknowledgements}

None.

\section{Financial support}

This research received no specific grant from any funding agency, commercial or not-for-profit sectors.

\section{Conflict of interest}

None.

\section{References}

Eisenberg L (1986). Mindlessness and brainlessness in psychiatry. British Journal of Psychiatry 148, 497-508.

Levav I, Saraceno B (2014). Primary prevention takes a leading role in world mental health action. European Journal of Psychiatry 28, 66-70.

Pariante CM (2015). Neuroscience, mental health and the immune system: overcoming the brain-mind-body trichotomy. Epidemiology and Psychiatric Sciences (this issue).

Rose N (2015). Neuroscience and the future for mental health? Epidemiology and Psychiatric Sciences (this issue).

Sijbrandij M, Kleiboer A, Bisson JI, Barbui C, Cuijpers P (2015). Pharmacological prevention of post-traumatic stress disorder and acute stress disorder: a systematic review and meta-analysis. Lancet Psychiatry 2, 413-421.

World Health Organization \& Gulbenkian Foundation (2014). Social determinants of mental health. World Health Organization: Geneva. Retrieved from: http://apps.who.int/ iris/bitstream/10665/112828/1/9789241506809_eng.pdf?ua=1 